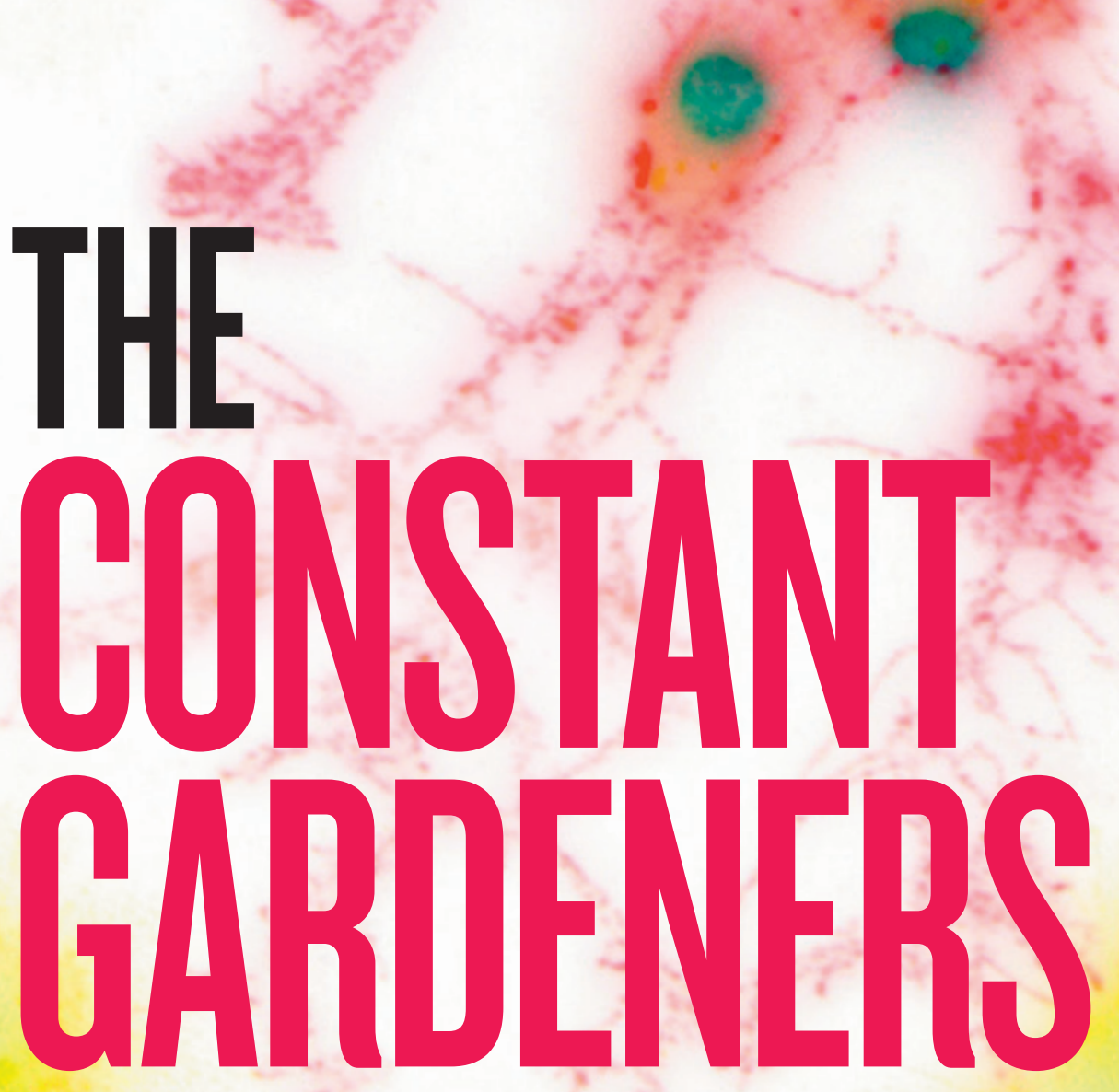

ONCE THOUGHT TO BE PASSIVE SENTINELS, MICROGLIA NOW SEEM TO BE CRUCIAL FOR PRUNING BACK NEURONS DURING DEVELOPMENT.

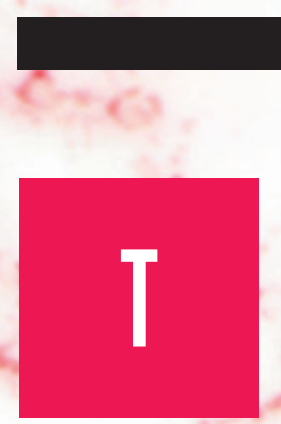

BY VIRGINIA HUGHES spider-like resting state, but only in slices of dead tissue. No one had been able to see them in a live brain. That's because microglia - which, unlike other cells in the brain, are part of the immune system - are extremely sensitive. Cut a nerve, or release infectious bacteria into brain tissue, and microglia spring into action, retracting their many appendages and morphing into big, round blobs that gobble up pathogens and clear away cellular wreckage. 
To see the cells without disturbing them, Nimmerjahn used a newly published approach for imaging a live mouse brain ${ }^{1}$. After anaesthetizing the animal and peeling back its scalp, he removed the top twothirds of the skull's thickness and shaved the bone down to just 20 micrometres - thin enough for light to penetrate, but thick enough to avoid setting off the microglia. The work progressed slowly — Nimmerjahn had to douse the surgical site with cooling fluid after realizing that even the heat from scraping could aggravate the cells. But within a few months at the bench, he was able to record some time-lapse movies $^{2}$.

He was floored by what he saw: 'resting' microglia are anything but. Their delicate branches snake through densely packed brain tissue, constantly extending and shrinking and re-growing. "They're very dynamic, much more than any other cell in the adult brain," says Nimmerjahn, now a biophysicist at the Salk Institute in La Jolla, California. He calculated that the cells' concerted movements could survey the entire brain every couple of hours. But it was unclear why the microglia were moving so much, Nimmerjahn says. "Why does the brain invest so much energy?"

\section{CODED SIGNALS}

Nimmerjahn is not the only researcher intrigued by this question. A flurry of studies during the past two years - the latest published last week in Neuron ${ }^{3}$ - have investigated microglia's influence in adult and developing brains. The results are overturning the idea that microglia are passive immune sentinels. Several groups have proposed that these shape-shifting cells not only eat up invaders and damaged tissue, but also trim away weak connections, or synapses, between neurons. This pruning process occurs on a large scale in the developing brain, and it is known to be important in learning and memory. Neurodevelopmental disorders such as autism and schizophrenia are often associated with faulty pruning. Two provocative studies have suggested that mouse models of obsessivecompulsive disorder ${ }^{4}$ and Rett syndrome ${ }^{5}$, an autism spectrum disorder, show marked improvements after their microglia are replaced.

But many questions remain. No one knows how microglia 'talk' to neurons or other cells, or whether their functions are limited to certain brain regions, notes Richard Ransohoff, a neurologist at the Cleveland Clinic in Ohio and a co-author of the latest Neuron report. But, he adds, that makes the field ripe for discovery. "The possible ramifications of these observations, for both developmental diseases like autism and degenerative diseases like Alzheimer's, are virtually endless."

For more than a century, neurons have been the stars of neuroscience. But they account for only about $10 \%$ of the cells in the human brain. The balance is made up by different types of glia, which surround and support neurons and influence neuronal signalling. Oligodendrocytes, for example, create fatty sheaths that encase long neuronal branches and help to speed up electrical impulses. Astrocytes surround synapses and have been shown to affect neuronal signalling by controlling the mix of chemical messengers at neuronal junctions.

Microglia are quite different from their neighbours. Unlike neurons and other glial cells, they begin in the embryonic yolk sac as immunecell progenitors, just as the macrophages that patrol the bloodstream for foreign invaders do. During prenatal development - within eight embryonic days in a mouse - microglia migrate to the brain, where they become its dedicated immune cells. Researchers assume that the brain needs microglia because the blood-brain barrier seals it off not only from toxins, pathogens and some drugs in the bloodstream, but also from the immune cells circulating there.

Microglia spring into action in most brain diseases, engulfing pathogens, dead cells and misfolded proteins. They also clear away synapses that have been damaged by injury. It is not a big intellectual leap, then, to suppose that microglia have similar roles in the healthy brain, says Helmut Kettenmann, a neurobiologist at the Max Delbrück Center for Molecular Medicine in Berlin, Germany, who has studied glia for the past 30 years. Given the spate of new imaging studies, he says, "I think microglia cells are probably extremely important for synapse remodelling and plasticity in development."

The idea is gaining traction. At the annual meeting of the Society for Neuroscience last November, when some 30,000 neuroscientists convened in Washington DC, the organizers held a session on microglia for the first time. It was packed. The society later circulated a list of the ten most-searched terms on the meeting's website. 'Microglia' was number six, just behind dopamine, optogenetics and schizophrenia.

The momentum has been building since April 2005, when Nimmerjahn published his movies ${ }^{2}$. A month later, a team led by Wen-Biao Gan - a neuroscientist at New York University, who first developed the skull-thinning method - published similar results". "This was a major breakthrough and inspired a lot of people," says Marie-Eve Tremblay, a postdoctoral researcher at the University of Wisconsin-Madison who studies the role of microglia in sleep and wakefulness.

In 2010, Tremblay and her colleagues published a report on the activity of microglia in the visual cortex of young mice. In rodents and in people, this region is known for its plasticity: the animal begins life with a large number of synapses, and then the ones that are not activated by light input from the eyes are gradually pruned away. Tremblay's study showed that microglia seem to interact with small synapses that disappear within a couple of days. Moreover, when she housed the animals temporarily in darkness - effectively dampening neural activity in the visual cortex - microglia changed into their active, blobby form and seemed to hug synapses more snugly ${ }^{7}$.

Around the same time, other scientists were watching microglia in another highly plastic region of the developing mouse brain - the hippocampus, which is important for learning and memory. They examined young mice lacking the fractalkine receptor protein, which is expressed only by microglia and which binds fractalkine, a protein present on neurons. The mutant mice have an abundance of weak and immature synapses in the hippocampus, according to a study ${ }^{8}$ published last September. By the time the mutant mice are adults, the number of synapses normalizes, but the researchers have unpublished data showing that some other synaptic problems remain.

"My guess is that there's probably a very close signalling going on between neurons and microglia, back and forth, that coordinates which synapses they prune," says Cornelius Gross, a neuroscientist at the European Molecular Biology Laboratory in Monterotondo, Italy, who led the study. The trouble is that none of these studies indicate what that signal is. "What you'd like to find is some sort of an 'eat-me' marker, like in Alice in Wonderland, displayed on synapses that are to be pruned," Gross says. The latest report in Neuron claims to have identified such a tag ${ }^{3}$.

\section{FULL COMPLEMENT}

The study's roots date back to 2007, when Ben Barres, a neurobiologist at Stanford University in California, together with postdoc Beth Stevens and their colleagues, showed that pruning in a deep area of the visual system - part of the thalamus called the lateral geniculate nucleus, or LGN - depends on certain proteins in the complement cascade, part of the innate immune system that helps to clear out pathogens and unwanted cells. The researchers reported that complement proteins are expressed by immature neuronal cells and are more likely to show up around immature synapses than elsewhere during key periods of brain development. Mice that lack complement proteins show a mess of unrefined neural connections ${ }^{9}$. 
According to Barres, all of this suggested that the complement system was responsible for tagging weak synapses for pruning. But how could this tagging lead to synapse removal? "Our obvious hypothesis was that it might work exactly the same way as it works in the immune system," Barres says. In the bloodstream, complement proteins tag harmful bacteria, signalling for macrophage cells to come along and eat them. Microglia are both the brain's resident macrophages and the only brain cells that express the complement receptor. Nimmerjahn, who happened to be starting a postdoc with Barres at the time, had already shown that microglia were interacting with synapses in the mature brain. But nobody knew anything about what microglia were doing in the young brain.

Stevens focused on that question beginning in late 2008, when she launched her own lab at Boston Children's Hospital in Massachusetts. The Neuron paper is her team's first publication. Dori Schafer, a postdoc in Stevens's lab, designed an assay for imaging the LGN using a previously developed mutant mouse whose microglia glow bright green under ultraviolet light.

Schafer further altered the mice so that the synapses connected to one eye would appear red, and those linked to the other eye, blue. She then used chemicals to increase the firing of neurons in one eye and decrease it in the other eye. Because neuronal activity is known to make synapses stronger, this manipulation is thought to weaken the synapses for one eye and strengthen those for the other.

\section{RIGHT TIME, RIGHT PLACE}

The researchers' colourful pictures, showing fragments of red and blue synapses in the bellies of the microglia, suggested that the cells selectively engulf the weakest synapses (see picture). "This was really exciting because it showed for the first time that, $\mathrm{OK}$, the microglia are actually sensing, in some way, activity changes in the neuron," Schafer says.

The researchers then repeated the experiments using mice that lacked the complement receptor. Their theory predicted that getting rid of the receptor would thwart microglia's ability to eat up complementtagged synapses. Sure enough, these mutant animals had microglia with significantly less-full bellies.

Despite such findings, neuroscientists are still debating whether microglia have an active role in synaptic pruning. The pretty pictures put the cells at the right time and place, but no one has caught them in the act. "We can't see microglia picking off a synapse in real time," Schafer says. The cells could be initiating the operation, or they may simply swoop in to clear up the carnage after the synapse has been destroyed.

It is also unclear whether neuronal activity influences the complement-tagging process, notes Carla Shatz, a neurobiologist at Stanford. Her work has shown that other immune-system players the molecules of the major histocompatibility complex - are necessary for synaptic pruning, influenced by neuronal activity and likely to show up near complement proteins ${ }^{10}$. "I think there's a way to connect all of these observations, and to really test the idea that they're all part of a signalling system for synapse elimination," Shatz says. But current microscopy tools are a limiting factor. "We just don't have the resolution to see which cells are making the molecules versus which ones are receiving the signals."

Meanwhile, microglia studies continue to yield unexpected results. A 2010 study of mouse models of trichotillomania - a psychiatric disorder characterized by compulsive hair-pulling - showed that disruption of the Hoxb8 gene, which in the brain is expressed only by certain types of microglia, caused mice to groom the fur right off their bodies ${ }^{4}$. The behaviour stopped after the mice received wholebody irradiation followed by a bone-marrow transplant. The researchers speculate that radiation wipes out defective microglia (and the blood-brain barrier) and allows them to be replaced with new ones from the transplanted bone marrow.

Similarly, a study published in March showed that a bone-marrow transplant greatly increased the lifespan of mouse models of Rett syndrome ${ }^{5}$.

\section{SINGULAR POWER}

These studies have raised the alluring idea that microglial manipulations could be used to treat neurodevelopmental disorders. "This is the only population in the brain that could be very easily replaced," says Jonathan Kipnis, a neuroscientist at the University of Virginia in Charlottesville and lead investigator of the Rett study. "If we learn how to harness microglia for the benefit of the brain, I think clinically that would be a powerful tool."

The transplant papers have many critics. It is not known, for one thing, how many microglia repopulate the brain after the procedure. And wiping out an animal's immune system is bound to cause a host of changes. But Stevens, Gross, Tremblay and others are all forging ahead, beginning to investigate the role of microglia in various mouse models of autism.

Researchers are also eager to get their hands on a much-anticipated tool: mutant mice that have genes knocked out of microglia but not other cells. Such 'conditional-knockout' mice can be used to investigate a gene's function specifically in microglia, as opposed to in astrocytes or other cells.

Gan says that he and his team have already created conditionalknockout mice for two genes - MECP2, which is mutated in human cases of Rett syndrome, and GRN, which has mutations associated with a heritable form of frontotemporal dementia. The results are not yet published.

"Right now, the excitement is good about microglia," Gan says. "We still need to do the hard work to figure out what exactly they do."

\footnotetext{
Virginia Hughes is a freelance science writer in New York City.

1. Grutzendler, J., Kasthuri, N. \& Gan, W. B. Nature 420, 812-816 (2002).

2. Nimmerjahn, A., Kirchhoff, F. \& Helmchen, F. Science 308, 1314-1318 (2005)

3. Schafer, D. P. et al. Neuron 74, 691-705 (2012).

4. Chen, S.-K. et al. Cell 141, 775-785 (2010).

5. Derecki, N. C. et al. Nature 484, 105-109 (2012).

6. Davalos, D. et al. Nature Neurosci. 8, 752-758 (2005).

7. Tremblay, M.-Ë., Lowery, R. L. \& Majewska, A. K. PLoS Biol. 8, e1000527 (2010)

8. Paolicelli, R. C. et al. Science 333, 1456-1458 (2011)

9. Stevens, B. et al. Cell 131, 1164-1178 (2007)

10. Datwani, A. et al. Neuron 64, 463-470 (2009)
} 